\title{
Rayleigh-Ritz Variational Approximation and Symmetry Nonrestoration
}

\author{
Giovanni AMELINO-CAMELIA \\ Theoretical Physics, Oxford University, 1 Keble Rd., Oxford OX1 3NP, UK
}

\begin{abstract}
The investigation of symmetry nonrestoration scenarios has led to a controversy, with certain nonperturbative approximation schemes giving indications in sharp disagreement with those found within conventional perturbation theory. A Rayleigh-Ritz variational approach to the problem, which might be useful in bridging the gap between perturbative and nonperturbative viewpoints, is here proposed. As a first application, this approach is used in the investigation of a $Z_{2} \times Z_{2}$-invariant thermal field theory with two scalar fields, placing particular emphasis on the region of parameter space that has been claimed to support symmetry nonrestoration.
\end{abstract}

OUTP-96-44P hep-ph/9610262

July 1996 
The subject of temperature-induced phase transitions[1-3] in relativistic quantum field theories has been extensively investigated over the last twenty years. In particular, transitions from a high-temperature symmetric phase to a low-temperature phase in which some symmetries are spontaneously broken are a crucial ingredient in most modern cosmological scenarios, and have been shown to be realized in large classes of thermal field theories.

The possibility of symmetry nonrestoration (SNR) at high temperatures[3-7] (or transitions from a high-temperature broken-symmetry phase to a low-temperature symmetric phase [8]) could also have interesting phenomenological implications, most notably allowing to circumvent the monopole problem in certain Grand Unification Theories; however, the investigation of SNR scenarios has led to controversy, with certain nonperturbative approximation schemes giving indications in sharp disagreement with those found within conventional perturbation theory. Specifically, whereas perturbative analyses find that some models, possibly of phenomenological relevance, can support SNR upon appropriate (however ad hoc) choice of the available parameters, the corresponding analyses within certain nonperturbative approximation schemes $\mid 9 \|$ indicate that symmetry is inevitably restored.

Recently, some progress has been made toward bridging the gap between perturbative and nonperturbative results on SNR. This has been attained via the use of improved perturbative techniques [10, 11] in which, while preserving the general structure of the perturbative expansion, some nonperturbative features of the theory are effectively taken into account. The preliminary results of this improved perturbative approaches have indicated [10, 11] that the conventional (unimproved) perturbative techniques overestimate the "SNR parameter space" (the region of parameter space capable of supporting SNR), and it is reasonable to interpret these results as suggesting that further improvements in the accuracy of the approximations would ultimately lead to the conclusion that the SNR parameter space is actually empty, just as predicted within the nonperturbative approximation schemes adopted in Ref. [9]. This expectation is encouraged by the findings of related studies on the lattice[12].

In this Letter, I discuss techniques which can be useful in making further progress in the direction proposed in the Refs. [10, 11], and, as a first application, which also serves as illustrative example, I use them in the investigation of the two-scalar-field theory of Euclidean Lagrange density

$$
L=\frac{1}{2}\left(\partial_{\mu} \Phi\right)\left(\partial^{\mu} \Phi\right)+\frac{1}{2}\left(\partial_{\mu} \Psi\right)\left(\partial^{\mu} \Psi\right)+\frac{1}{2} m^{2} \Phi^{2}+\frac{1}{2} \omega^{2} \Psi^{2}+\frac{\lambda_{\Phi}}{24} \Phi^{4}+\frac{\lambda_{\Psi}}{24} \Psi^{4}-\frac{\lambda_{\Phi \Psi}}{4} \Phi^{2} \Psi^{2},
$$

which is $Z_{2} \times Z_{2}$ invariant $[(\Phi \rightarrow-\Phi) \times(\Psi \rightarrow-\Psi)]$, and is among the strongest candidates as a model supporting SNR.

Within conventional perturbation theory the model (1) is found to support SNR when円

$$
\lambda_{\Phi}<\lambda_{\Phi \Psi}<\sqrt{\lambda_{\Phi} \lambda_{\Psi}}
$$

or

$$
\lambda_{\Psi}<\lambda_{\Phi \Psi}<\sqrt{\lambda_{\Phi} \lambda_{\Psi}} .
$$

The lower bound on $\lambda_{\Phi \Psi}$ in the above (2) and (3) is set by the requirement that, in the high temperature limit, one of the fields have imaginary dressed (thermal) mass, which within perturbation theory is found to be given by $M \sim \sqrt{\left(\lambda_{\Phi}-\lambda_{\Phi \Psi}\right) T^{2}}$ and $\Omega \sim \sqrt{\left(\lambda_{\Psi}-\lambda_{\Phi \Psi}\right) T^{2}}$ respectively for $\Phi$ and $\Psi$. (Symmetry is not restored if the square of the dressed mass, i.e. the second derivative of the effective potential at the origin, is negative.) The common upper

\footnotetext{
${ }^{1}$ Note that here and in the following $\lambda_{\Phi}$ and $\lambda_{\Psi}$ are assumed to be positive, so that there is at least a chance of having a stable theory, and all the $\lambda$ 's are assumed to be small, so that perturbation theory has at least a chance of giving consistent results.
} 
bound on $\lambda_{\Phi \Psi}$ given in (2) and (3) follows from (naive 11]) stability analysis [3]. However, in light of the improved perturbative analyses of Refs. 110, 11] it appears that conventional perturbation theory underestimates the lower bound of the SNR parameter space, since the inclusion of higher order effects shows [10, 11] that the condition $\lambda_{\Phi}<\lambda_{\Phi \Psi}$ (or equivalently $\left.\lambda_{\Psi}<\lambda_{\Phi \Psi}\right)$ is not sufficient to render one of the dressed masses imaginary, and might also overestimate the upper bound of the $S N R$ parameter space, since the higher-order analysis of ultraviolet structures indicates [1] that the condition $\lambda_{\Phi \Psi}<\sqrt{\lambda_{\Phi} \lambda_{\Psi}}$ is necessary but not sufficient for stability.

While clearly a lot could be learned by further extending/improving the analyses presented in Refs. 10, 11], this objective is presented with serious technical hurdles, primarily as a result of the necessity to study complicated self-consistent equations for the dressed masses (which pick up a momentum dependence beyond leading order). In this Letter, I use a variational approximation to estimate the leading correction to the results of Refs. 110, 11. Specifically, I am interested in the way this leading correction modifies the result for the lower bound of the SNR parameter space. If a more stringent bound was found, one would be presented with a scenario in which each next step of improvement of the analysis appears to lead to a smaller $S N R$ parameter space, pointing toward the validity of the above mentioned conjecture that the SNR parameter space is actually empty. However, as reported below, I find that instead the leading correction to the result of Refs. [10, 11] on the lower bound of the SNR parameter space is in favour of SNR. This suggests that additional steps of improvement of the analysis might lead to alternating contributions, ultimately resulting in a lower bound of the SNR parameter space which does not differ much from the one obtained in Refs. 10, 11], and therefore is still consistent with SNR in the $Z_{2} \times Z_{2}$ model under consideration.

Since the analysis here presented is purely concerned with the lower bound of the $S N R$ parameter space, my result does not affect the possibility that the $S N R$ parameter space might be found to be empty once the issues of stability (which are relevant for the upper bound of the SNR parameter space) raised in Ref. [11] are fully investigated. This however appears to require several nontrivial steps, starting with the identification (within a study of the type reported in Ref.[13] for the $Z_{2}$ model) of the conditions rigorously necessary (and sufficient) for the stability of the $Z_{2} \times Z_{2}$ model. This challenging program is left for future work.

In preparation for the derivation of the announced result on the lower bound of the SNR parameter space, let me start by revisiting the results of Refs. [10, 11]. I do this in the framework of the CJT formalism of the effective potential for composite operators[11,1416], which is ideally suited for a variational analysis of the type I discuss here. I choose to work in the imaginary time formalism of finite temperature field theory, wherein the fourth component of momentum is discretized, $k_{4}=i \pi n T$ ( $n$ even for bosons), and I adopt the notation

$$
\oint_{k} \equiv T \sum_{n=-\infty}^{\infty} \int \frac{d^{3} k}{(2 \pi)^{3}} .
$$

I also exploit the fact that the two SNR scenarios emerging within conventional perturbation theory, (2) and (3), are simply related by renaming of fields and couplings, and therefore, for the purpose of the analysis here presented, it is sufficient to consider $\lambda$ 's such that

$$
\lambda_{\Phi}<\lambda_{\Phi \Psi}<<\lambda_{\Psi}
$$

which allows to investigate the lower bound of the SNR parameter space in the candidate SNR scenario (2). This is useful since (5) implies [11] that all the interesting structures of the effective potential $V(\phi, \psi)$, corresponding to the shifts $\{\Phi, \Psi\} \rightarrow\{\Phi+\phi, \Psi+\psi\}$, are

\footnotetext{
${ }^{2}$ Clearly, the setup (5) implies that at high temperatures the minimum of the effective potential along the $\psi$ direction is at $\psi=0$.
} 
to be found in its projection on the $\psi=0$ axis. Therefore, in the following I concentrate on $V(\phi, \psi=0)$, i.e. shifts $\{\Phi, \Psi\} \rightarrow\{\Phi+\phi, \Psi\}$, and, for short, I adopt the notation $V(\phi)$ for $V(\phi, \psi=0)$.

In Ref. 11 this potential $V(\phi)$ was investigated within the "bubble approximation", which is the lowest nontrivial order[14-18] of approximation of the effective potential in the CJT formalism. The result of Ref.[11] can be written, using the (renormalized) gap equations, as ${ }^{3}$

$$
\begin{aligned}
V= & \frac{m^{2}}{2} \phi^{2}+\frac{\lambda_{\Phi}}{24} \phi^{4}+Q[M]+Q[\Omega]+\left(\frac{m^{2}}{2}+\frac{\lambda_{\Phi}}{4} \phi^{2}-\frac{M^{2}}{2}\right) P[M] \\
& +\left(\frac{\omega^{2}}{2}+\frac{\lambda_{\Phi \Psi}}{4} \phi^{2}-\frac{\Omega^{2}}{2}\right) P[\Omega]-\frac{\lambda_{\Phi}}{8}(P[M])^{2}-\frac{\lambda_{\Psi}}{8}(P[\Omega])^{2}+\frac{\lambda_{\Phi \Psi}}{4} P[M] P[\Omega],
\end{aligned}
$$

where $M$ and $\Omega$ are the dressed masses of the fields $\Phi$ and $\Psi$ respectively, and are determined by the gap equations

$$
\begin{aligned}
M^{2} & =m^{2}+\frac{\lambda_{\Phi}}{2} \phi^{2}+\frac{\lambda_{\Phi}}{2} P[M]-\frac{\lambda_{\Phi \Psi}}{2} P[\Omega], \\
\Omega^{2} & =\omega^{2}+\frac{\lambda_{\Phi \Psi}}{2} \phi^{2}+\frac{\lambda_{\Psi}}{2} P[\Omega]-\frac{\lambda_{\Phi \Psi}}{2} P[M] .
\end{aligned}
$$

In the above (6)-(8), $P$ is the "tadpole" [2]

$$
P[X] \equiv \oiint_{p} \frac{1}{k^{2}+X^{2}}=\frac{X^{2}}{16 \pi^{2}} \ln \frac{X^{2}}{\mu^{2}}-\int \frac{d^{3} k}{(2 \pi)^{3}}\left[\sqrt{|\mathbf{k}|^{2}+X^{2}}\left(1-\exp \left(\frac{\sqrt{|\mathbf{k}|^{2}+X^{2}}}{T}\right)\right)\right]^{-1}
$$

and $Q$ is the "one loop" [2]

$$
Q[X]=\oiint_{k} \ln \left[k^{2}+X^{2}\right]=\frac{X^{4}}{64 \pi^{2}}\left[\ln \frac{X^{2}}{\mu^{2}}-\frac{1}{2}\right]+T \int \frac{d^{3} k}{(2 \pi)^{3}} \ln \left[1-\exp \left(\frac{\sqrt{|\mathbf{k}|^{2}+X^{2}}}{T}\right)\right] .
$$

By stopping at the level of the bubble approximation used in Refs. 111 (and effectively used in Refs. 10 although the formalism adopted there is different) one ends up ignoring contributions to the second derivative with respect to $\phi$ of the effective potential at the origin (i.e. the square of the dressed mass of the field $\Phi$ at $\phi=0$ ) which are of order $\lambda^{2} \ln \lambda$. Taking this into account, and using the following known[2] "high temperature" (small $X / T$ ) expansions

$$
\begin{gathered}
P[X] \simeq \frac{T^{2}}{12}-\frac{X T}{4 \pi}-\frac{X^{2}}{16 \pi^{2}} \ln \frac{X^{2}}{T^{2}}, \\
Q[X] \simeq-\frac{\pi^{2} T^{4}}{90}+\frac{X^{2} T^{2}}{24}-\frac{X^{3} T}{12 \pi}-\frac{X^{4}}{64 \pi^{2}} \ln \frac{X^{2}}{T^{2}},
\end{gathered}
$$

\footnotetext{
${ }^{3}$ Since, for the reasons discussed above, I am considering only the dependence on higher order terms of the lower bound of the SNR parameter space, which is quite decoupled[11] from the issues of stability and ultraviolet structure, for brevity my analysis here does not provide details on renormalization (which the reader can find in Ref.|11]), and also ignores the small cut-off dependent contribution to the effective potential that is present when the theory, as appropriate for a trivial theory 11, 15, is considered with a finite cut-off[11].
} 
one finds that the Eqs.(6)-(8) lead to the following result for the second derivative of the effective potential at the origin

$$
V^{\prime \prime}(0) \simeq m^{2}+\left(\lambda_{\Phi}-\lambda_{\Phi \Psi}\right) \frac{T^{2}}{24}+\lambda_{\Phi \Psi} \sqrt{\lambda_{\Psi}-\lambda_{\Phi \Psi}} \frac{T^{2}}{16 \sqrt{6} \pi},
$$

where indeed terms of order $\lambda^{2} \ln \lambda$ and higher have been dropped, and the relations (5) have been used in extracting the leading contribution of order $\lambda \sqrt{\lambda}$. Notice that, in the scenario (5), the contribution to (13) of order $\lambda$, which can be estimated in conventional (unimproved) perturbation theory, is negative, i.e. works in favour of SNR, but the contribution of order $\lambda \sqrt{\lambda}$, whose evaluation requires to take into account the bubble diagrams[11], is positive. Most importantly the positivity of the correction of order $\lambda \sqrt{\lambda}$ renders insufficient for SNR the condition $\lambda_{\Phi \Psi}>\lambda_{\Phi}$, which sets the lower bound of the SNR parameter space within conventional perturbation theory. This is the basis for the recent observation 10, 11] that the bubble-improved perturbation theory predicts a smaller SNR parameter space than conventional perturbation theory.

The improvement in the determination of the SNR parameter space that I report in this Letter relies on an estimate of the leading correction to (13), which is of order $\lambda^{2} \ln \lambda$ (notice that $|\epsilon|^{2}<|\epsilon|^{2} \ln |\epsilon|<|\epsilon|$ for small $\epsilon$ ). I obtain this result via the use of variational techniques in the framework of the CJT formalism, which allows to go systematically[11,14-16] beyond the bubble approximation. In the CJT formalism the effective potential $V$ is obtained as the solution of a variational problem for the effective potential for composite operators $W$ :

$$
\begin{gathered}
V(\phi)=W[\phi ; M(\phi ; k)] \\
{\left[\frac{\delta W[\phi ; \mathcal{M}(k)]}{\delta \mathcal{M}(k)}\right]_{\mathcal{M}(k)=M(\phi ; k)}=0,}
\end{gathered}
$$

A rigorous definition of $W$ can be found in Refs.[14-17], but for the purposes of the present Letter it is sufficient to observe that $W$ admits a loop expansion, with $\mathcal{M}(k)$ appearing as the (dressed) mass matrixf:

$$
W=V_{\text {tree }}(\phi)+\frac{1}{2} \oint_{k} \operatorname{Tr} \ln \left[k^{2}+\mathcal{M}^{2}\right]+\frac{1}{2} \oint_{k} \frac{m_{\text {tree }}^{2}-\mathcal{M}^{2}}{k^{2}+\left[\mathcal{M}^{2}(k)\right]}+W^{*}[\phi ; \mathcal{M}(k)],
$$

where $V_{\text {tree }}(\phi)$ is the tree-level (classical) potential, $m_{\text {tree }}$ is the tree-level mass, and $W^{*}$ is given by all the two-particle-irreducible 14] vacuum-to-vacuum graphs with two or more loops in the theory with vertices given by the interaction part of the shifted $(\Phi \rightarrow \Phi+\phi)$ Lagrangian and propagator set equal to $G(k)$, with $\left[G^{-1}(k)\right]_{a b}=\delta_{a b} k^{2}+\left[\mathcal{M}^{2}(k)\right]_{a b}$.

The bubble approximation, which is the lowest nontrivial order[14-18] of approximation of the effective potential in the CJT formalism, is obtained by including in $W^{*}$ only the "doublebubble diagrams", i.e. diagrams made of two rings touching at one point. Importantly, the contributions of double-bubble diagrams have the form

$$
V_{\infty}(X, Y)=\oint_{k} \oint_{p} \frac{1}{k^{2}+X^{2}} \frac{1}{p^{2}+Y^{2}}=P[X] P[Y]
$$

\footnotetext{
${ }^{4}$ In the present Letter the formalism is set up in terms of the dressed masses, whereas in Ref. [11] it was set up in terms of the dressed propagators. The two setups are equivalent (if no assumption is made concerning the momentum dependence of the dressed objects), but the discussion in terms of the dressed masses guides more directly toward the type of Rayleigh-Ritz approximation to be discussed later.
} 
which does not involve any flow of momenta from one loop to the other. The possibility to make substantial analytic progress, ultimately leading to the result (6), is a peculiarity of the bubble approximation, which in particular involves dressed masses that are exactly momentum independent [11, 18]. As soon as one goes beyond the bubble approximation the analysis becomes much more complicated. The dominant higher-order contributions, already relevant at the order $\lambda^{2} \ln \lambda$ here under consideration, come from "sunset" diagrams

$$
V_{\ominus}(X, Y, Z)=\oiint_{k} \oiint_{p} \frac{1}{k^{2}+X^{2}} \frac{1}{p^{2}+Y^{2}} \frac{1}{(k+p)^{2}+Z^{2}}
$$

which arise from the contraction of two copies of any three-point vertex present in the shifted $(\{\Phi, \Psi\} \rightarrow\{\Phi+\phi, \Psi\})$ Lagrangian. In these diagrams momentum does flow from one loop to the other leading to the loss of all simplifications encountered in the bubble approximation. In such cases, analytic progress requires that the effective potential be evaluated approximately. The fact that in the CJT formalism the effective potential is obtained as the solution of a variational problem renders available the "machinery" of variational approximations. In particular, one can resort to approximations of the Rayleigh-Ritz type 14, 18, in which, rather than considering arbitrary variations of the dressed masses $\mathcal{M}_{a b}(k)$, one takes a parameter-dependent ansatz for $\mathcal{M}_{a b}(k)$ and vary only the parameters. This variational approach was recently used in Ref. 18 in a related study of gauge theories at finite temperature, and has been well received 19, 20], although it is hard 18, 19 to establish its range of validity in the framework of the ("thermally troublesome") gauge theories. The non-gaugetheoretical model considered in the present Letter is, however, closer to the contexts in which Rayleigh-Ritz variational approaches have been traditionally used, and one can expect the usual arguments] for its reliability to hold. Therefore, a Rayleigh-Ritz variational approach can be useful in investigations of the issue of restoration/nonrestoration of symmetry in the $Z_{2} \times Z_{2}$ model, and, as a first step in that direction, I use it here in estimating the leading non-bubble contribution to the effective potential, which comes from the sunset diagrams.

Before proceeding with this estimate, let me observe that within the bubble approximation one could have already resorted to a Rayleigh-Ritz approximation. In fact, as the reader can easily check, by substituting in the bubble-approximated CJT effective potential for composite operators the following variational ansatz for the dressed masses

$$
\mathcal{M}_{a b}=\delta_{a 1} \delta_{b 1} M+\delta_{a 2} \delta_{b 2} \Omega
$$

one obtains a Rayleigh-Ritz bubble effective potential which, after stationarizing with respect to the (dimensionful) parameters $M$ and $\Omega$, reproduces Eq.(6) within the accuracy achievable in the bubble approximation, with $M$ and $\Omega$ identified with the solutions of (17)-(8). In fact this is encoded in the fact that the bubble effective potential obtained in Ref. [11] is stationary (again up to terms which are however not reliably determined by the bubble approximation) with respect to variations of $M$ and $\Omega$ in the neighborhood of the solutions of the gap equations (ब)-(8). In this sense the Rayleigh-Ritz variational approach is exact within the

\footnotetext{
${ }^{5}$ The variational problem in which the CJT formalism casts the evaluation of the effective potential is well suited 18] for exact numerical analysis even when non-bubble contributions are included, although, as a consequence of triviality, some care is needed in the handling of ultraviolet structures.

${ }^{6}$ The reader should keep in mind that there are two levels of approximation involved in the analysis presented in this Letter. To begin with, I am working within truncations/approximations of the effective potential that arise within the formalism of the loop expansion of the CJT effective potential. When this loop expansion is truncated at the "bubble level" the calculation can be completed without further approximation. If one goes beyond the bubble approximation, to the "sunset level", even the truncated expression of the CJT effective potential cannot be analyzed exactly (unless numerical techniques are developed), and a further variational approximation is needed to estimate analytically the sunset-truncated CJT effective potential.

${ }^{7}$ Discussion of these topics can be found in Ref. 21.
} 
bubble approximation, as it should be expected since the variational ansatz (19) is just in the form of the exact (bubble-approximated) dressed mass matrix.

In order to estimate the contribution of the sunset diagrams within the Rayleigh-Ritz approach it is sufficient to take the following three steps. First, add the relevant sunset terms to the bubble-approximated CJT effective potential. Then, evaluate the resulting expression for the effective potential with a physical parameter-dependent ansatz for the dressed masses. Finally, stationarize with respect to the parameters characterizing the ansatz.

Clearly in this higher-order analysis the result of the Rayleigh-Ritz approach is not exact, but, through the variational procedure, it should provide a reliable 21] way to encode into a handleable set of parameters the bulk of the effect of the sunset-induced momentum dependence of the dressed masses. Actually, as a result of the observation above concerning the exactness of the ansatz (19) at the bubble level, one can expect that the ansatz (19) be sufficient also to estimate the leading sunset correction; in fact, as long as the bubble contributions lead over the sunset ones (i.e. at small values of the coupling constants) the dressed masses will be only weakly momentum dependent, so that it should be possible to replace them with momentum independent "effective masses" to be determined self-consistently.

Adopting the ansatz (19) one finds that the handling of sunset diagrams only requires to evaluate $V_{\ominus}(X, Y, Z)$ with momentum independent $X, Y$, and $Z$. This has already been done in Refs. [22, 23], where, in particular, it was shown that the leading high temperature contribution, which is of interest for my analysis, is given by

$$
V_{\ominus}(X, Y, Z) \simeq \frac{T^{2}}{16 \pi^{2}} \ln \left(\frac{3 T}{X+Y+Z}\right)
$$

From Eq.(16) one can easily show that the complete "sunset-approximated" (i.e. obtained by adding the contributions from the sunset diagrams to the bubble-approximated effective potential) CJT effective potential can be written as

$$
\begin{aligned}
V= & \frac{m^{2}}{2} \phi^{2}+\frac{\lambda_{\Phi}}{24} \phi^{4}+Q[M]+Q[\Omega]+\left(\frac{m^{2}}{2}+\frac{\lambda_{\Phi}}{4} \phi^{2}-\frac{M^{2}}{2}\right) P[M] \\
& +\left(\frac{\omega^{2}}{2}+\frac{\lambda_{\Phi \Psi}}{4} \phi^{2}-\frac{\Omega^{2}}{2}\right) P[\Omega]+\frac{\lambda_{\Phi}}{8}(P[M])^{2}+\frac{\lambda_{\Psi}}{8}(P[\Omega])^{2}-\frac{\lambda_{\Phi \Psi}}{4} P[M] P[\Omega] \\
& -\frac{\lambda_{\Phi}^{2}}{12} \phi^{2} V_{\ominus}(M, M, M)-\frac{\lambda_{\Phi \Psi}^{2}}{4} \phi^{2} V_{\ominus}(\Omega, \Omega, M) .
\end{aligned}
$$

As discussed above, the ordinary effective potential is obtained from this formula by stationarizing with respect to variations of $M$ and $\Omega$. Then, also using the high temperature expansions (11), (12), and (20), with some simple algebra one can show that the sought contribution 8 of order $\lambda^{2} \ln \lambda$ to the second derivative of the effective potential at the origin is given by

$$
\begin{gathered}
\frac{\lambda_{\Phi \Psi} \Omega^{2}}{16 \pi^{2}} \ln \left(\frac{\Omega}{T}\right)-\frac{\lambda_{\Phi} M^{2}}{16 \pi^{2}} \\
\ln \left(\frac{M}{T}\right)+\frac{\lambda_{\Phi \Psi}^{2} T^{2}}{16 \pi^{2}} \ln \left(\frac{2 \Omega+M}{3 T}\right)+\frac{\lambda_{\Phi}^{2} T^{2}}{48 \pi^{2}} \ln \left(\frac{\Omega}{T}\right) \simeq \\
\simeq \frac{\lambda_{\Phi \Psi} \Omega^{2}}{16 \pi^{2}} \ln \left(\frac{\Omega}{T}\right) \simeq-\frac{\lambda_{\Phi \Psi} \lambda_{\Psi} T^{2}}{768 \pi^{2}} \ln \frac{24}{\lambda_{\Psi}}<0,
\end{gathered}
$$

where the indicated approximations hold in the scenario (5) presently under consideration, which also implies $M<<\Omega$.

\footnotetext{
${ }^{8}$ Terms of order $\lambda^{2} \ln \lambda$ are already present in the bubble approximation (these terms are among those listed in Eq.(22)), but could not be included 10 in the bubble approximation of the second derivative of the effective potential at the origin, given in Eq.(13), since they are not reliably determined when the sunset contributions are not taken into account.
} 
Eq.(22) shows that, as anticipated in the opening of this Letter, the leading $O\left(\lambda^{2} \ln \lambda\right)$ correction to Eq.(13) is negative, i.e. works in favour of SNR. This is certainly of encouragement for the possibility of SNR, with the proviso about stability mentioned in the opening of this Letter. It should also be noticed that the SNR-favouring correction here identified is quite small, especially as a result of the numerical prefactor, and, while possibly sufficient to help the case of SNR in the toy model here considered, it might be negligible in the context of models relevant for particle physics, where large gauge-coupling contributions render SNR very unlikely [10]. The Rayleigh-Ritz variational approach here advocated should be useful in further investigation of these issues.

It is a pleasure to acknowledge conversations with G. Bimonte, R. Jackiw, G. Lozano, O. Philipsen, S.-Y. Pi, and S. Sarkar. 


\section{References}

[1] D. Kirzhnits, JETP Lett. 15 (1972) 529; D. Kirzhnits and A. Linde, Phys. Lett. B42 (1972) 471; D. Kirzhnits and A. Linde, JETP 40 (1974) 628.

[2] L. Dolan and R. Jackiw, Phys. Rev. D 9 (1974) 3320.

[3] S. Weinberg, Phys. Rev. D9 (1974) 3357.

[4] R.N. Mohapatra and G. Senjanovic, Phys. Rev. Lett. 42 (1979) 1651; Phys. Rev. D20 (1979) 3390.

[5] G. Dvali, A. Melfo, and G. Senjanovic, Phys. Rev. Lett. 75 (1995) 4559.

[6] T. G. Roos, hep-th/9511073.

[7] G. Dvali, A. Melfo, and G. Senjanovic, hep-ph/9601376; G. Dvali and K.Tamvakis, hep-ph/9602336.

[8] P. Langacker and S.-Y. Pi, Phys. Rev. Lett. 45 (1980) 1.

[9] Y. Fujimoto and S. Sakakibara, Phys. Lett. B151 (1985) 260; E. Manesis and S. Sakakibara, Phys. Lett. B157 (1985) 287; G.A. Hajj and P.N. Stevenson, Phys. Rev. D413 (1988) 413; K.G.Klimenko, Z.Phys. C43 (1989) 581; Theor. Math. Phys. 80 (1989) 929.

[10] G. Bimonte and G. Lozano, Phys. Lett. B366 (1996) 248; Nucl. Phys. B460 (1996) 155.

[11] G. Amelino-Camelia, Nuc. Phys. B476 (1996) 255.

[12] G. Bimonte and G. Lozano, hep-th/9603201.

[13] W.A. Bardeen and Moshe Moshe, Phys. Rev. D28 (1983) 1372.

[14] J.M. Cornwall, R. Jackiw, and E. Tomboulis, Phys. Rev. D10, 2428 (1974).

[15] G. Amelino-Camelia and S.-Y. Pi, Phys. Rev. D47 (1993) 2356.

[16] R. Jackiw and G. Amelino-Camelia, hep-ph/9311324, in Proceedings of the Third Workshop on Thermal Field Theories and Their Applications, Banff, Canada, August 15-27, 1993, edited by F.C. Khanna, R. Kobes, G. Kunstatter, and H. Umezawa (World Scientific, 1994).

[17] P. Castorina, M. Consoli, and D. Zappala, Phys. Lett. B201 (1988) 90.

[18] G. Amelino-Camelia, Phys. Rev. D49 (1994) 2740.

[19] J.R. Espinosa, M. Quiros, and F. Zwirner, Phys. Lett. B314 (1993) 206.

[20] K. Farakos, K. Kajantie, K. Rummukainen, and M. Shaposhnikov, Nucl. Phys. B425 (1994) 67.

[21] H.A. Bethe and R. Jackiw, Intermediate Quantum Mechanics, third ed. (The Benjamin/Cummings Publishing Company, Menlo Park, California, 1986).

[22] R.R. Parwani, Phys. Rev. D45 (1992) 4695.

[23] P. Arnold and O. Espinosa, Phys. Rev. D47 (1993) 3546. 